\title{
AUTOEXAMEN DE SENO, COMO PREVENCIÓN EN LAS ADOLESCENTES DE COLEGIOS PÚBLICOS Y PRIVADOS DE LA CIUDAD DE NEIVA EN EL 2009
}

\author{
Breast self-examination as prevention in adolescents from public \\ and private high schools in neiva in 2009
}

Fecha de recibido: 11-08-2010 • Fecha de aprobación: 20-10-2010

\begin{abstract}
Resumen. La detección temprana del cáncer de seno es de vital importancia en la supervivencia y pronóstico de esta patología, por lo tanto nuestro objetivo fundamental es identificar si las adolescentes de formación básica secundaria conocen y realizan adecuadamente el autoexamen de seno.
\end{abstract}

Se realizaron 1000 encuestas que constaban de diecisiete preguntas, y fueron realizadas en estudiantes de los grados 9,10 y 11 de colegios públicos y privados de la ciudad de Neiva Huila, Colombia, entre septiembre y diciembre de 2009.

Teniendo en cuenta, las estadísticas del Departamento Administrativo Nacional de Estadística (DANE), la población aproximada entre los 14 y 18 años (edad en la cual se está en formación básica secundaria), es de 32.000 adolescentes en la ciudad de Neiva, luego, con previo muestreo se realiza una encuesta donde se adopta un error admisible del 0,0816\% y un índice de confianza del 99\%.

De la población encuestada, el 71\% afirmó conocer el autoexamen de seno, sin embargo, sólo un 37\% lo realizan, y el 15,5\% del total lo practican en forma adecua- da un $11,1 \%$, examina sus senos una semana después de la menstruación, el 18,1\% lo efectuaban mensualmente, el $65,5 \%$ del total no lo realizaba porque no saben cómo practicar la técnica, no obstante, el 99,1\% de las adolescentes están interesadas en realizar el autoexamen de seno.

La educación impartida por los padres, las instituciones educativas, las entidades y el personal de salud, se considera deficiente, teniendo en cuenta que las adolescentes no conocen el autoexamen de seno.

Palabras clave: medicina del adolescente, cáncer de seno, autoexamen de seno.

Abstract. Early detection of breast cancer is of paramount importance for survival and prognosis of this pathology. This study aims mainly at identifying whether adolescents of basic secondary education know and carry out breast self-examination in a proper way.

1000 surveys consisting of seventeen questions were administered to 9 th, 10 th and 11th graders from public

\footnotetext{
* Estudiantes Programa de Medicina, Facultad de salud, Universidad Surcolombiana.

$\ddagger$ Correo electrónico: johanmcp@hotmail.com

** Cirujano Mastólogo, Departamento de Cirugía Hospital Universitario de Neiva, Profesor Asistente, Departamento de Ciencias Clínicas, Universidad Surcolombiana.
} 
and private high schools in Neiva-Huila, Colombia, between September and December, 2009.

Based on the statistical data from the Departamento Administrativo Nacional de Estadistica (DANE), the approximate population between 14 and 18 years old (basic secondary education age), is of 32.000 adolescents in Neiva. Then, after selecting a sample, a survey is administered with permissible error of $0.0816 \%$ and a confidence rate of $99 \%$.

From the surveyed sample, $71 \%$ know breast selfexamination; nevertheless, just $37 \%$ use the technique appropriately, $11,1 \%$ do breast self-examination a week after menstruation, $18,1 \%$ do it once in a month, $65,5 \%$ do not do it because of lack of technique. However, $99 \%$ of adolescents are interested in carrying out breast self-examination.

Education coming from parents, schools, governmental institutions and health care givers is poor since adolescents do not know breast self-examination.

Key words: adolescent medicine, breast cancer, breast self-examination.

\section{INTRODUCCIÓN}

El cáncer de seno constituye actualmente uno de los principales problemas en salud pública a nivel mundial, teniendo en cuenta que es la neoplasia maligna más diagnosticada y la mayor causa de mortalidad por cáncer en el sexo femenino(1).

Según estudios, su incidencia se ha extendido considerablemente en los últimos 10 años, pues se estima que para el año 2010 habrían alrededor de 1.450 .000 casos nuevos; lo que define un aumento aproximado al $82 \%$ con respecto a lo estimado en $1990^{(2,3)}$.

La incidencia es mayor en países desarrollados donde es hasta 6 veces más frecuente que en países en vía de desarrollo(4,5).

En Colombia, las publicaciones reportan que es el segundo cáncer más frecuente en mujeres; en los departamentos de Antioquia, Arauca, Atlántico, Bolívar y Valle, genera hasta el 23,3\% de las muertes por cáncer, y en Bogotá es la decimocuarta causa de muerte en general ${ }^{(6)}$.

Esta realidad se repite en Colombia y en el departamento del Huila donde según estadísticas institucionales de la unidad de cáncer del Hospital Universitario Hernando Moncaleano Perdomo de Neiva, se presentan aproximadamente 140 casos nuevos por año, en su gran mayoría en estados avanzados y en pacientes jóvenes en edad productiva económica y socialmente ${ }^{(7)}$.

Por lo tanto, el autoexamen de seno, práctica realizada por la mujer, mensualmente, siguiendo una técnica establecida, es de trascendental importancia para la detección precoz de cáncer de seno, como ha sido demostrado por los estudios en San Petersburgo y Shangai ${ }^{(8)}$. El autoexamen de seno no debe considerarse como único método de tamizaje, encaminado a la detección temprana del cáncer. Dependiendo de la edad de la mujer la mamografía presenta una mayor utilidad después de los 50 años ${ }^{(9)}$.

Asimismo, se ha evidenciado una marcada asociación entre la falta de práctica del autoexamen de seno y retraso en el diagnostico de neoplasias malignas en la glándula mamaria ${ }^{(5,9)}$ y de manera similar, en la práctica clínica en el Hospital Universitario Hernando Moncaleano Perdomo de Neiva, en el área de Mastología, se ha identificado que la mayoría de las pacientes con cáncer de seno, no realizaban la técnica del autoexamen de seno.

A pesar de ser una enfermedad detectable en la fase inicial pocas son las mujeres que dan importancia a los diferentes métodos de prevención como el autoexamen, la consulta anual rutinaria al médico y la mamografía.

En adición, el perfil socio cultural de la región está cargado de tabúes religiosos y de desconocimiento del propio cuerpo, lo que hace que las pacientes busquen servicios de salud en fase avanzada de la enfermedad, en un estudio inicial sobre frecuencia de cáncer en el Hospital Universitario Hernando Moncaleano Perdomo el $70 \%$ de las pacientes consultó en un estadio avanzado de la enfermedad ${ }^{(8)}$.

La morbilidad y la mortalidad por cáncer de seno pueden reducirse si las mujeres tienen conocimiento, la motivación y la destreza necesaria para aplicar medidas de detección precoz como es el autoexamen de seno. El referido cuidado debe ser iniciado en la adolescencia y practicarlo a través de su vida.

Los objetivos radican en evaluar el conocimiento y práctica del autoexamen de seno en adolescentes de básica secundaria. Identificar la relación entre la realización del autoexamen de 
seno y estrato socioeconómico de las entrevistadas. Establecer el motivo de la no realización del autoexamen de seno. Identificar factores que inciden directa o indirectamente en la correcta práctica del autoexamen de seno, concientizar a la población femenina de la importancia del autoexamen de seno.

\section{MATERIALES Y MÉTODOS}

El trabajo se inició, realizando una invitación personal por parte de los autores y una comunicación formal escrita por parte del profesor del área (cirujano mastólogo), a los rectores de 10 colegios públicos y privados de la ciudad de Neiva, algunos solo femenino y otros de conformación mixta existió la aceptación verbal en 6 colegios (3 públicos y 3 privados).

Los colegios con orientación religiosa, no aceptaron la invitación.

Se realizó un muestreo previo de 20 encuestas, 10 en el Colegio Liceo Santa Librada y 10 en el Colegio Rafael Pombo, en los grados 9, 10 y 11 con el objeto de ajustar el formato de las encuestas que se debían realizar en la población que se estudiaría y calcular el tamaño de la muestra.

El resultado del muestreo, fue 50\% conocían y realizaban el autoexamen de seno y el 50\% no conocían y por lo tanto no lo realizaban.

Según la estimación del DANE para el 2010, el total de habitantes del área urbana de la ciudad de Neiva son 310.000 de ellas un $52,6 \%$ son mujeres y de estas el 19,62\% tienen entre 14 y 18 años, es decir aproximadamente 32.000 mujeres adolescentes.

El cálculo de la muestra se realizó mediante la aplicación de la siguiente fórmula(7):

$$
\mathrm{n}=\mathrm{P}(100-\mathrm{P}) \alpha^{2} /(\mathrm{P} . \mathrm{d})^{2}
$$

\section{Donde}

$\mathrm{n}=$ número de encuestas a realizar.

$\mathrm{P}=$ porcentaje hallado en muestreo previo $=50 \%$.

$\alpha=$ factor que determina el grado de confianza. $1=68 \% ; 1,96=95 \% ; 2,58=99 \%$.

$\mathrm{d}=$ error admisible. $0,1=10 \%$ de error admisible; $0,2=20 \%$ de error admisible.

Adoptamos un error admisible del 0,816\% y un índice de confianza de $99 \%$.

$\mathrm{n}=50(100-50) 2,58^{2} /\left(100^{*} 0.0816\right)^{2}=999,7$

encuestas.
Es un estudio epidemiológico analítico, observacional, transversal, y se realizó en 1000 estudiantes de los grados 9, 10 y 11, de los colegios públicos (Colegio Santa Librada, Colegio Liceo Santa Librada, Colegio INEM Julián Motta Salas) y privados (Colegio Rafael Pombo, Colegio IDHEA, Colegio Heidelberg) de la ciudad de Neiva Huila, durante el periodo comprendido entre el 1 de septiembre al 9 de diciembre de 2009.

Los datos se obtuvieron de 1000 encuestas anónimas cuyo interrogatorio consistía en 17 preguntas de las cuales dos eran sobre información personal, dos para identificar si conocían y realizaban la técnica del autoexamen de senos, nueve se referían a la metodología de la técnica realizada, una para identificar dónde adquirieron la información para realizar la técnica, y tres de opinión personal.

De las 17 preguntas, dos eran abiertas y 15 cerradas. Dichas encuestas fueron realizadas por los autores.

La encuesta permitió recolectar toda la información necesaria para obtener los resultados esperados (figura 1).

Complementariamente posterior a la encuesta se les entrego a cada de las participantes un plegable educativo diseñado por los autores donde se describía la técnica adecuada del autoexamen de seno y hábitos de vida saludables.

\section{RESULTADOS}

Se analizaron 1000 encuestas realizadas en colegios públicos y privados de la ciudad de Neiva, encontrando los siguientes hallazgos.

La totalidad de las adolescentes encuestadas, estaban en un rango de edad entre los 12 y 20 años de edad, siendo la mayoría entre 14 y 16 años $(71 \%)$ y entre los estratos socioeconómicos 1 y $3(93 \%)$. El 71\% responde saber sobre el autoexamen de seno, sin embargo el $63 \%$ de las encuestadas afirman no examinar sus senos (Tablas 1, 2).

Con respecto al nivel educativo el grado noveno con 412 y décimo con 358 estudiantes, en un 36\% refiere que se realizan el autoexamen de seno y un $64 \%$ no se lo realizan. El grado once, con 230 alumnas, el $40 \%$ responde que se examina los seno y el $60 \%$ no los examina. 


\section{AUTOEXAMEN DE SENO}

Edad:

Estrato: 1__2__3__4__5__6__

Lea cuidadosamente cada pregunta y marque con una $X$ su respuesta:

1. ¿Conoce cómo se realiza el autoexamen de seno? SI _

2. ¿Examina frecuentemente sus senos? $\mathrm{SI} \_\mathrm{NO}$

SI SU RESPUESTA ES NO CONTINÚE CON LA PREGUNTA 14, DE LO CONTRARIO SIGA CON LA PREGUNTA 3

3. En relación a su menstruación, ¿cuándo examina sus senos?

Una semana antes ___ Durante___ Una semana después__ Indiferentemente__

4. ¿Cada cuánto lo realiza? Semanal__ Mensual__ Trimestral__ Semestral__Anual__

5. ¿Qué técnica utiliza? Observación__ Palpación__ Ambas__

6. ¿En qué posición observa sus senos? Sentada__ Parada_ Ambas__

7. ¿En qué posición palpa sus senos? Parada__ sentada_ Acostada_

8. ¿Con qué parte de la mano, palpa sus senos?

Con toda la mano__ Con los dedos _ Con la yema de los dedos_

9. ¿Cómo examina los senos? Ambas a la vez De a una por vez

10. Cuando examina sus senos, ¿incluye el examen del pezón? SI_ NO

11. Para examinar el seno derecho utiliza:

La mano derecha__ La mano izquierda_ Ambas manos _ Solo la observa

12. ¿De dónde obtuvo la información para realizar el autoexamen?

Libros / folletos__ TV__ Radio__ Internet__ Médico__ Familiares

13. ¿Por qué no lo realiza?

Porque no sabe cómo__ Porque no le interesa__ Por temor_No lo considera necesario

14. ¿A qué edad considera conveniente comenzar el autoexamen?

15. ¿Considera que el autoexamen de los senos es importante? SI__ NO__

Figura 1. Autoexamen de seno, como prevención en las adolescentes de colegios públicos y privados de la ciudad de Neiva en el 2009. 
Tabla 1. Porcentaje de conocimiento de autoexamen de seno en población adolescente femenina de instituciones públicas y privadas de la ciudad de Neiva.

\begin{tabular}{ccc}
\hline $\begin{array}{c}\text { Conoce el auto } \\
\text { examen }\end{array}$ & Frecuencia & Valor (\%) \\
\hline Si & 290 & 29 \\
No & 710 & 71 \\
Total & 1000 & 100 \\
\hline
\end{tabular}

Tabla 2. Porcentaje de realización de autoexamen de seno en población adolescente femenina de instituciones públicas y privadas de la ciudad de Neiva.

\begin{tabular}{ccc}
\hline $\begin{array}{c}\text { Examina } \\
\text { sus senos }\end{array}$ & Frecuencia & Valor (\%) \\
\hline $\mathrm{Si}$ & 370 & 37 \\
$\mathrm{No}$ & 630 & 63 \\
Total & 1000 & 100 \\
\hline
\end{tabular}

Con respecto al adecuado tiempo y forma para realizarse el autoexamen de seno de las 370 adolescentes que se realizan el autoexamen el 20\% se lo realiza antes del ciclo menstrual, el 30\% después del ciclo, el 55\% durante la menstruación y el 35\% indiferentemente.

De las adolescentes que se realizan el autoexamen el 33\% lo realiza con una frecuencia semanal, el 49\% mensual, el 6\% trimestral, el 5\% lo efectúa semestral y el $7 \%$ lo realiza anualmente.

De las 370 de las jóvenes al responder la pregunta que si siguen alguna técnica, 267 de ellas, es decir, el $72 \%$ siguen alguna técnica y el $28 \%$ respondió no seguir ninguna técnica.

De las 267 estudiantes que dijeron seguían alguna técnica el $14,6 \%$ se realizan el autoexamen solo con observación, un $20,9 \%$ solo con palpación y un $64,5 \%$ realizan observación y palpación en el autoexamen.

De las 370 estudiantes que se realizan el autoexamen obtuvieron la información como se describe (tabla 3).

De las estudiantes que se realizan el autoexamen, cuando se valora en las preguntas la forma como lo realizan: posición, forma de observación y técnica de palpación, se encuentra que el 57,89\% no realiza bien el autoexamen y el $42,11 \%$ lo rea-
Tabla 3. Origen de la información para la realización del autoexamen de seno en población adolescente femenina de instituciones públicas y privadas de la ciudad de Neiva.

\begin{tabular}{ccc}
\hline $\begin{array}{c}\text { Fuente de } \\
\text { información }\end{array}$ & Frecuencia & Valor (\%) \\
\hline Libros / folletos & 67 & 18 \\
TV & 84 & 23 \\
Radio & 0 & 0 \\
Internet & 11 & 3 \\
Médico & 104 & 28 \\
Familiares & 104 & 28 \\
Total & 370 & 100 \\
\hline
\end{tabular}

liza adecuadamente (155 adolescentes del total de las 1.000).

$\mathrm{Al}$ analizar las 630 estudiantes que manifestaron que no se realizan el autoexamen de seno, el $65,2 \%$ indicaron porque no saben cómo hacerlo, el 3,6\% por qué no les interesa, el $9,5 \%$ por temor, y un $21,7 \%$ no lo considera necesario.

En cuanto a la edad para comenzar la realización del autoexamen, en el grupo que lo realiza el $71,2 \%$ afirmó que antes de los 15 años y el grupo que no se lo realiza el $61,2 \%$ considera que se lo deben iniciar después de los 15 años.

En cuanto a la pregunta de la importancia de la realización del autoexamen el 99,1\% lo consideran significativo y el 0,9 consideran que no.

\section{DISCUSIÓN}

No se encontraron publicaciones específicas que hagan referencia a la práctica del autoexamen de seno en mujeres en esta etapa de su vida, por lo tanto no existe una forma comparativa real entre nuestros resultados y los resultados de otras publicaciones.

Sin embargo, un estudio publicado en Jordania, realizado en estudiantes universitarias de pre y postgrado y empleados universitarios, acerca de los factores y las creencias que pueden estar relacionados con la práctica del auto-examen del seno. Los resultados indican que aunque la mayoría de la población de la muestra $(67 \%)$ había oído/leído sobre el autoexamen de seno, sólo una cuarta parte de ellas informó que se había practicado alguna vez la técnica en los últimos 12 
meses, y sólo el 7\% la había realizado de forma regular mensual ${ }^{(10)}$.

Los resultados obtenidos en Jordania son similares a los hallados en nuestro estudio, a pesar que los grupos etarios de los dos estudios son diferentes; puesto que, sólo el $15,5 \%$ de las adolescentes, practican adecuadamente el autoexamen de seno y en Jordania sólo el 7\% de las mujeres indagadas realizaban la técnica de la manera apropiada.

Como bien se sabe que el autoexamen de seno es fundamental en la prevención secundaria del cáncer de seno ${ }^{(11)}$, en la actualidad no se está enseñando en las adolescentes una etapa de la vida en la cual la mujer se encuentra en formación y sería fundamental la enseñanza del mismo.

A pesar de que existe una noción general de que es, en qué consiste y para qué sirve el autoexamen de seno, se hace necesario por parte del personal docente y del personal de salud, consolidar los conceptos básicos en esta población, para garantizar la realización del autoexamen con una técnica adecuada.

Una de las tareas que debemos profundizar en el aspecto educativo es capacitar a los docentes o en su defecto al personal de salud primario para que dedique tiempo en la enseñanza de la técnica en los colegios públicos y privados.

\section{REFERENCIAS}

1. Carvalho Fernández, A.F. 2006. Práctica del autoexamen de mama por usuarias del Sistema Único de Salud de Ceará. 2006. Revista Cubana de Enfermería 22(3).

2. World Health Organization. The world Health Report: Changing history (France). En: www.who.int/whr/2004/en/report04_ en.pdf. 2004. Consulta: noviembre de 2010.
3. Dershaw, D.D. Mammography current status and high risk screening. 30 conferencia sobre cáncer de mama. Dallas, 2002. Citado por: Examen de mamas, medicina efectiva contra el cáncer. Cordero Otelo D. Ecos de Mantua 73.

4. Boyle, Peter. 2005. Breast cancer control: signs of progress, but more work required. Breast 14(6):429-438.

5. Abdel-Fattah, M., Zaki, A., Bassili, A., Health, J. 2000. Breast self-examination practice and its impact on breast cancer diagnosis in Alexandria, Egypt. East Mediterranean Health Journal 6(1):34-40.

6. Piñeros, M., Hernandez, G. 2004. Bray F Increasing mortality rates of common malignancies in Colombia: an emerging problem. Cancer 101(10):2285-2292.

7. Gutiérrez, A.M., Olaya, J.G., Medina, R. 2009. Frecuencia de cáncer de seno mediante detección temprana en el hospital universitario de Neiva entre el 1 de junio y el 30 de noviembre de 2007. Revista Colombiana de Cirugía 24:31-8.

8. Pascua, M. 1974. Metodología Bioestadística para médicos y oficiales sanitarios. Madrid: Editorial Paz Montalvo 338-340.

9. Semiglazov, V.F., Sagaidak, V.N., Moiseyenko, V.M. et al. 1993. Study of the role of breast self-examination in the reduction of mortality from breast cancer. The Russian Federation/ World Health Organization Study. European Journal of Cancer 29(14):2039-2046.

10. Petro-Nustus, W., Blanche, I.M. 2002. Factors Associated with Breast Self-Examination Among Jordanian Women. Public Health Nursing 19(4):263-271.

11. Harvey, B.J., Miller, A.B., Baines, C. 1997. Effect of breast self-examination techniques on the risk of death from breast cancer. Canadian Medical Association Journal 157:1205-1212. 